\title{
Online Embedding Compression for Text Classification Using Low Rank Matrix Factorization
}

\author{
Anish Acharya, ${ }^{* 1}$ Rahul Goel, ${ }^{1}$ Angeliki Metallinou, ${ }^{1}$ Inderjit Dhillon ${ }^{2,3}$ \\ ${ }^{1}$ Amazon Alexa AI, ${ }^{2}$ Amazon Search Technologies, ${ }^{3}$ University of Texas at Austin \\ \{achanish, goerahul, ametalli\}@amazon.com, isd@a9.com
}

\begin{abstract}
Deep learning models have become state of the art for natural language processing (NLP) tasks, however deploying these models in production system poses significant memory constraints. Existing compression methods are either lossy or introduce significant latency. We propose a compression method that leverages low rank matrix factorization during training, to compress the word embedding layer which represents the size bottleneck for most NLP models. Our models are trained, compressed and then further re-trained on the downstream task to recover accuracy while maintaining the reduced size. Empirically, we show that the proposed method can achieve $90 \%$ compression with minimal impact in accuracy for sentence classification tasks, and outperforms alternative methods like fixed-point quantization or offline word embedding compression. We also analyze the inference time and storage space for our method through FLOP calculations, showing that we can compress DNN models by a configurable ratio and regain accuracy loss without introducing additional latency compared to fixed point quantization. Finally, we introduce a novel learning rate schedule, the Cyclically Annealed Learning Rate (CALR), which we empirically demonstrate to outperform other popular adaptive learning rate algorithms on a sentence classification benchmark.
\end{abstract}

\section{Introduction}

Deep learning has achieved great success in various NLP tasks such as sequence tagging (Chung et al. 2014), (Ma and Hovy 2016) and sentence classification (Liu et al. 2015; Kim 2014). While traditional machine learning approaches extract hand-designed and task-specific features, which are fed into a shallow model, neural models pass the input through several feedforward, recurrent or convolutional layers of feature extraction that are trained to learn automatic feature representations. However, deep neural models often have large memory-footprint and this poses significant deployment challenges for real-time systems that typically have memory and computing power constraints. For example, mobile devices tend to be limited by their CPU speed, memory and battery life, which poses significant size constraints for models embedded on such devices. Similarly,

\footnotetext{
${ }^{*}$ Corresponding Author Copyright (C) 2019, Association for the Advancement of Artificial Intelligence (www.aaai.org). All rights reserved.
}

models deployed on servers need to serve millions of requests per day, therefore compressing them would result in memory and inference cost savings.

For NLP specific tasks, the word embedding matrix often accounts for most of the network size. The embedding matrix is typically initialized with pretrained word embeddings like Word2Vec, (Mikolov et al. 2013) FastText (Bojanowski et al. 2016) or Glove (Pennington, Socher, and Manning 2014) and then fine-tuned on the downstream tasks, including tagging, classification and others. Typically, word embedding vectors are 300 -dimensional and vocabulary sizes for practical applications could be up to 1 million tokens. This corresponds to up to $2 \mathrm{Gbs}$ of memory and could be prohibitively expensive depending on the application. For example, to represent a vocabulary of $100 \mathrm{~K}$ words using 300 dimensional Glove embeddings the embedding matrix would have to hold $60 \mathrm{M}$ parameters. Even for a simple sentiment analysis model the embedding parameters are $98.8 \%$ of the total network (Shu and Nakayama 2017).

In this work, we address neural model compression in the context of text classification by applying low rank matrix factorization on the word embedding layer and then retraining the model in an online fashion. There has been relatively less work in compressing word embedding matrices of deepNLP models. Most of the prior work compress the embedding matrix offline outside the training loop either through hashing or quantization based approaches (Joulin et al. 2016; Shu and Nakayama 2017; Raunak 2017).

Our approach includes starting from a model initialized with large embedding space, performing a low rank projection of the embedding layer using Singular Value Decomposition (SVD) and continuing training to regain any lost accuracy. This enables us to compress a deep NLP model by an arbitrary compression fraction $(p)$, which can be pre-decided based on the downstream application constraints and accuracy-memory trade-offs. Standard quantization techniques do not offer this flexibility, as they typically allow compression fractions of $1 / 2$ or $1 / 4$ (corresponding to 16-bit or 8-bit quantization from 32-bits typically).

We evaluate our method on text classification tasks, both on the benchmark SST2 dataset and on a proprietary dataset from a commercial artificial agent. We show that our method significantly reduces the model size (up to 90\%) with minimal accuracy impact (less than 2\% relative), and outper- 
forms popular compression techniques including quantization (Hubara et al. 2016) and offline word embedding size reduction (Raunak 2017).

A second contribution of this paper is the introduction of a novel learning rate schedule,we call Cyclically Annealed Learning Rate (CALR), which extends previous works on cyclic learning rate (Smith 2017) and random hyperparameter search (Bergstra and Bengio 2012). Our experiments on SST2 demonstrate that for both DAN (Iyyer et al. 2015) and LSTM (Hochreiter and Schmidhuber 1997) models CALR outperforms the current state-of-the-art results that are typically trained using popular adaptive learning like AdaGrad. Overall, our contributions are:

- We propose a compression method for deep NLP models that reduces the memory footprint through low rank matrix factorization of the embedding layer and regains accuracy through further finetuning.

- We empirically show that our method outperforms popular baselines like fixed-point quantization and offline embedding compression for sentence classification.

- We provide an analysis of inference time for our method, showing that we can compress models by an arbitrary configurable ratio, without introducing additional latency compared to quantization methods.

- We introduce CALR, a novel learning rate scheduling algorithm for gradient descent based optimization and show that it outperforms other popular adaptive learning rate algorithms on sentence classification.

\section{Related Work}

In recent literature, training overcomplete respresentations is often advocated as overcomplete bases can transform local minima into saddle points (Dauphin et al. 2014) or help discover robust solutions (Lewicki and Sejnowski 2000). This indicates that significant model compression is often possible without sacrificing accuracy and that the model's accuracy does not rely on precise weight values (Keskar et al. 2016). Most of the recent work on model compression exploits this inherent sparsity of overcomplete represantations. These works include low precision computations (Anwar, Hwang, and Sung 2015; Courbariaux, Bengio, and David 2014) and quantization of model weights (Han, Mao, and Dally 2015; Zhou et al. 2017). There are also methods which prune the network by dropping connections with low weights (Wen et al. 2016; See, Luong, and Manning 2016) or use sparse encoding (Han et al. 2015). These methods in practice often suffer from quantization loss especially if the network is deep due to a large number of low-precision multiplications during forward pass. Quantization loss is hard to recover through further training due to the non-trivial nature of backpropagation in low precision (Lin et al. 2015). There has been some work on compression aware training (Polino, Pascanu, and Alistarh 2018) via model distillation (Hinton, Vinyals, and Dean 2015) that addresses this gap in accuracy. However, both quantized distillation and differentiable quantization methods introduce additional latency due to their slow training process that requires careful tuning, and may not be a good fit for practical systems where a compressed model needs to be rapidly tuned and deployed in production.

Low-rank matrix factorization (LMF) is a very old dimensionality reduction technique widely used in the matrix completion literature (see (Recht and Ré 2013) and references therein). However, there has been relatively limited work on applying LMF to deep neural models. (Sainath et al. 2013; Lu, Sindhwani, and Sainath 2016) used low rank matrix factorization for neural speech models. While (Sainath et al. 2013) reduces parameters of DNN before training, (Xue, Li, and Gong 2013) restructures the network using SVD introducing bottleneck layers in the weight matrices. However, for typical NLP tasks, introducing bottleneck layers between the deep layers of the model does not significantly decrease model size since the large majority of the parameters in NLP models come from the input word embedding matrix. Building upon this prior work, we apply LMF based ideas to reduce the embedding space of NLP models. Instead of exploiting the sparsity of parameter space we argue that for overcomplete representation the input embedding matrix is not a unique combination of basis vectors (Lewicki and Sejnowski 2000). Thus, it is possible to find a lower dimensional parameter space which can represent the model inputs with minimal information loss. Based on this intuition, we apply LMF techniques for compressing the word embedding matrix which is the size bottleneck for many NLP tasks.

\section{Methodology \\ 3.1 Low-rank Matrix Factorization (LMF) for Compressing Neural Models}

Low-rank Matrix Factorization (LMF) exploits latent structure in the data to obtain a compressed representation of a matrix. It does so by factorization of the original matrix into low-rank matrices. For a full rank matrix $W \in R^{m \times n}$ of rank $r$, there always exists a factorization $W=W_{a} \times W_{b}$ where $W_{a} \in \mathbb{R}^{m \times r}$ and $W_{b} \in \mathbb{R}^{r \times n}$. Singular Value Decomposition (SVD) (Golub and Reinsch 1970) achieves this factorization as follows:

$$
W_{m \times n}=U_{m \times m} \Sigma_{m \times n} V_{n \times n}^{t}
$$

where $\Sigma_{m \times n}$ is a diagonal rectangular matrix of singular values of descending magnitude. We can compress the matrix by choosing the $k$ largest singular values, with $k<m$ and $k<n$. For low rank matrices, the discarded singular values will be zero or small, therefore the following will approximately hold:

$$
W_{m \times n} \approx U_{m \times k} \Sigma_{k \times k} V_{k \times n}^{t}
$$

Therefore, the original matrix $W$ is compressed into two matrices:

$$
\begin{gathered}
W_{a}=U \in \mathbb{R}^{m \times k} \\
W_{b}=\Sigma_{k \times k} V_{k \times n}^{t} \in \mathbb{R}^{k \times n}
\end{gathered}
$$

The number of parameters are reduced from $m \times n$ to $k \times(m+n)$. Therefore, to achieve a $p$ fraction parameter reduction, $k$ should be selected as:

$$
\begin{array}{r}
(p \times m \times n)=k \times(m+n) \\
k=\left\lfloor\frac{p m n}{m+n}\right\rfloor ; k \in \mathbb{Z}^{+}
\end{array}
$$


The value $k$ can be varied depending on the desired compression fraction $p$ as long as $k \geq 1$. In practice, being able to decide an arbitrary compression fraction $p$ is an attractive property for model compression, since it allows choosing compression rates based on the accuracy-memory trade-offs of a downstream application. The low rank matrix factorization operation is illustrated in Figure 1, where a single neural network matrix (layer) is replaced by two low rank matrices (layers).

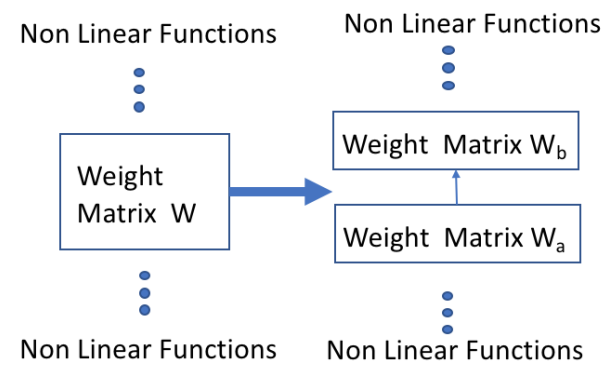

Figure 1: Replacing one neural network matrix with two low rank matrices

Compressing the Word Embedding Matrix For NLP tasks, typically the first layer of the neural model consists of an embedding lookup layer that maps the words into real-valued vectors for processing by subsequent layers. The words are indices $i$ of a word dictionary of size $D$ while the word embeddings are vectors of size $d$. This corresponds to a lookup table $E(i)=W_{i}$ where $W \in \mathbb{R}^{d \times|D|}$. The word embeddings are often trained offline on a much larger corpus, using algorithms like Word2Vec, GloVe etc, and are then fine-tuned during training on the downstream NLP task. Our method decomposes the embedding layer in an online fashion during training using eq.(5). $W_{a}$ becomes the new embedding layer and $W_{b}$ becomes the next layer. Continuing backpropagation over this new network struture finetunes the low rank embedding space and regains any accuracy loss within a few epochs.

\subsection{Cyclically Annealed Learning Rate}

Due to the non-convex nature of the optimization surface of DNNs, gradient based algorithms like stochastic gradient descent (SGD) are prone to getting trapped in suboptimal local minima or saddle points. Adaptive learning rates like Adam (Kingma and Ba 2014) and Adagrad (Duchi, Hazan, and Singer 2011) try to solve this problem by tuning learning rate based on the magnitude of gradients. While in most cases they find a reasonably good solution, they usually can't explore the entire gradient landscape. (Bergstra and Bengio 2012; Loshchilov and Hutter 2016) indicates periodic random initialization or warm restarts often helps in better exploration of the landscape while (Smith 2017) showed that letting the learning rate(LR) vary cyclicaly (Cyclic learning rate(CLR)) helps in faster convergence. In this work we further refine CLR and propose a simulated annealing inspired LR update policy we call Cyclically Annealed Learning Rate (CALR). CALR is described in algorithm (1) and
Fig. 2b. In addition to varying LR in a CLR style triangular windows(Fig. 2a), CALR expontentially decays the upper bound $L R_{U B}$ of the triangular window from its initial value. When the learning rate decreases to a lower bound $L R_{L B}$, we increase the upper bound to its initial value $L R_{U B}$ and continue with the LR updates. This exponential decay ensures slow steps on the optimization landscape. Intuitively, the proposed warm restarts, motivated by simulated annealing, make CALR more likely to escape from local minima or saddle points and enables better exploration of the parameter space. CALR should have similar but less aggressive effect as random initialization or warm restarts of LR. While exponentially decayed small cyclic windows let CALR carefully explore points local to the current solution, cyclic temperature increase helps it jump out and explore regions around other nearby local minima. We verified this intuition empirically for our classification experiments (Table 1, Secton 5.4), where CALR was able to achieve further improvement compared to CLR and Adagrad.

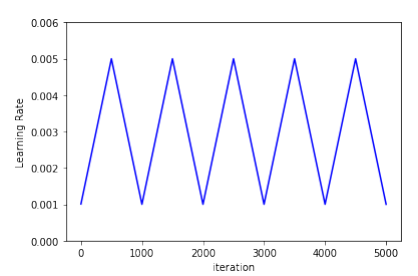

(a) CLR

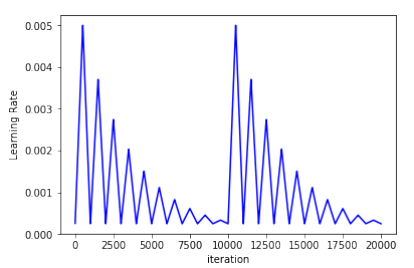

(b) CALR
Figure 2: Comparison of CLR and CALR update Policy

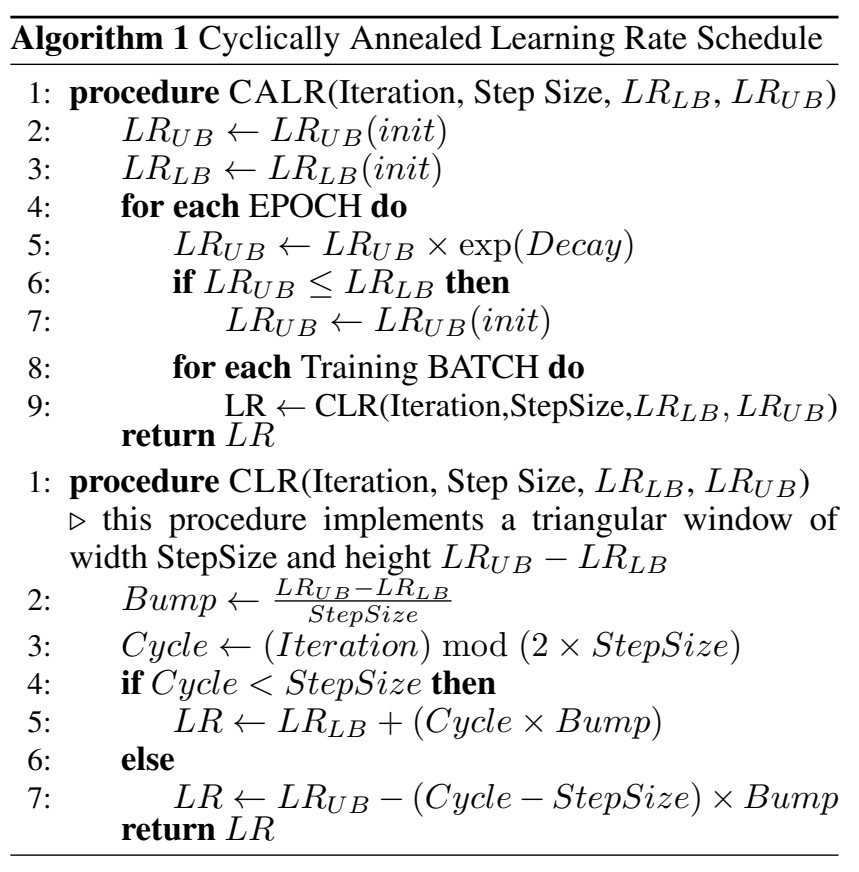

\subsection{Baselines}

We compare our proposed method with two commonly baselines for neural model compression. 
Fixed Point Weight Quantization (Baseline 1) We compare our approach with widely used post-training fixed point quantization (Bengio, Léonard, and Courville 2013; Gong et al. 2014) method where the weights are cast into a lower precision. This requires less memory to store them and doing low precision multiplications during forward propagation improves inference latency.

Offline Embedding Compression (Baseline 2) In this set of baseline experiments we compress the embedding matrix in a similar way using SVD but we do it as a preprocessing step similar to (Raunak 2017; Joulin et al. 2016). For example, if we have a 300 dimensional embedding and we want a $90 \%$ parameter reduction we project it onto a 30 dimensional embedding space and use this low dimensional embedding to train the NLP model.

\section{Analysis}

In this section we analyze space and latency trade-off in compressing deep models. We compare the inference time and storage space of floating point operations(FLOPs) between our method and Baseline1(sec.3.3) on a 1-layer Neural Network(NN) whose forward pass on input X can be represented as:

$$
f(X)=\sigma(X W)
$$

where $X \in \mathbb{R}^{1 \times m}$ and $W \in \mathbb{R}^{m \times n}$ and full rank. Our method reduces $\mathrm{W}$ into two low rank matrices $W_{a}$ and $W_{b}$ converting (6) into two layer NN represented as:

$$
f(X)=\sigma\left(\left(X \times W_{a}\right) \times W_{b}\right)
$$

where $W_{a} \in \mathbb{R}^{m \times k}$ and $W_{b} \in \mathbb{R}^{k \times n}$ are constructed as described in eq.(3) and eq.(4) choosing $\mathrm{k}$ as in eq.(5). Whereas, Baseline 1 casts the elements of $W$ to lower precision without restructuring the network. Thus, the quantized representation of a 1-layer NN is same as eq.(6) where $W_{i}$ is cast to a lower bit precision and eq. (7) is the corresponding representation in our approach where $W_{i}$ remains in its original precision.

\subsection{Space Complexity Analysis}

On Baseline1 each weight variable uses less number of bits for storage. Quantizing the network weights into low precision, we achieve space reduction by a fraction $\frac{B_{Q}}{B_{S}}$ where $B_{S}$ bits are required to store a full precision weight and $B_{Q}$ bits are needed to store low precision weight. In contrast, our approach described in section 3.1, achieves more space reduction as long as we choose $k$ (from eq (5)) such that:

$$
p<\frac{B_{Q}}{B_{S}}
$$

\subsection{Time Complexity Analysis}

Total number of FLOPs in multiplying two matrices (Trefethen and Bau III 1997) is given by: $F L O P(A \times B)=$ $(2 b-1) a c \sim O(a b c)$ where $A \in \mathbb{R}^{a \times b}, B \in \mathbb{R}^{b \times c}$ and $A B \in \mathbb{R}^{a \times c}$. Assuming one FLOP in a low precision matrix operations takes $T_{Q}$ time and on a full precision matrix it takes $T_{S}$ time, for the model structure given by eq. (6), we have: $a=1, b=m, c=n$. Hence, for the forward pass on a single data vector $X \in \mathbb{R}^{1 \times m}$ a quantized model uses $F_{Q}$ flops where:

$$
F_{Q}=(2 m-1) n
$$

Our algorithm restructures the DNN given in eq. (6) into eq. (7) yielding $F_{S}=F_{S 1}+F_{S 2}$ flops. where:

$$
\begin{array}{r}
F_{S 1}=(2 m-1) k, F_{S 2}=(2 k-1) n \\
F_{S}=2(m+n) k-(n+k)
\end{array}
$$

The number of FLOPs in one forward pass our method would be less than that of in Baseline1 if $F_{Q}<F_{S}$ or equivalently:

$$
\begin{aligned}
(2 m-1) n & >2(m+n) k-(n+k) \\
k & <\frac{2 m n}{2(m+n)-1}
\end{aligned}
$$

Under the reasonable assumption that $2(m+n) \gg 1$ or equivalently $2(m+n)-1 \approx 2(m+n)$, eq. (11) becomes:

$$
k<\frac{m n}{(m+n)}
$$

Eq. (12) will hold as long as $k$ in our method is chosen according to eq.(5) (since the fraction $p$ is by definition $p<1$ ) However, guaranteeing less FLOPs does not guarantee faster inference since quantized model requires less time per FLOP. Our method guarantees faster inference if the following condition holds:

$$
\begin{aligned}
F_{Q} T_{Q} & >F_{S} T_{S} \\
(2 m-1) n T_{Q} & >[(2 m-1) k+(2 k-1) n] T_{S} \\
\frac{(2 m-1) n}{[(2 m-1) k+(2 k-1) n]} & >\frac{T_{S}}{T_{Q}}
\end{aligned}
$$

where $T_{S}$ and $T_{Q}$ are time taken per FLOP for our method and Baseline1 respectivly. Again, we can make the reasonable assumptions that $2 m \gg 1$ and $2 k \gg 1$, therefore $2 m-1 \approx 2 m$ etc. Then eq. (13) becomes:

$$
\frac{m n}{(m+n) k}>\frac{T_{S}}{T_{Q}}
$$

Plugging $k$ from eq.(5) we have:

$$
p<\frac{T_{Q}}{T_{S}}
$$

If we assume that the time per FLOP is proportional to the number of bits $B$ for storing a weight variable, e.g., $T_{Q} \sim B_{Q}$ and $T_{S} \sim B_{S}$ then selecting $p$ such that $p<\frac{B_{Q}}{B_{S}}$ will ensure that eq. (15) holds. In most practical scenarios time saving is sublinear in space saving which means eq.(8) is necessary and sufficient to ensure our method has faster inference time than fixed point quantization(Baseline1).

\section{Experiments and Results}

Our experiments are focused on sentence classification task. 


\subsection{Datasets:}

For all the experiments reported we have used the following two sentence classification datasets:

Stanford Sentiment Treebank (SST2) SST2 (Socher et al. 2013) is a common text classification benchmark dataset that contains movie reviews, to be classified according to their sentiment. On an average the reviews are about 200 words long. The dataset contains standard train, dev, test splits and binary sentiment labels.

Books Intent Classification Dataset We use a proprietary dataset of around 60k annotated utterances of users interacting with a popular digital assistant about books related functionality. Each utterance is manually labeled with the user intent e.g., search for a book, read a book and others, with the task being to predict the user intent from a set of 20 intents. We choose $50 \mathrm{k}$ utterances as our training data, $5 \mathrm{k}$ as test data and $5 \mathrm{k}$ as dev data.

\subsection{Models:}

We evaluate our compression strategy on two types of widely used neural models:

Long Short Term Memory(LSTM) LSTMs, are powerful and popular sequential models for classification tasks. The LSTM units are recurrent inn nature, designed to handle long term dependencies through the use of input, output and forget gates and a memory cell. For input $X=$ $x_{1}, \cdots x_{T}$ and corresponding word embedding input vectors $e_{i}, i=1, \cdots T$, LSTM computes a representation $r_{t}$ at each word $t$, which is denoted as $r_{t}=\phi\left(e_{t}, r_{t-1}\right)$. Here, for sentence classification, we used the representation obtained from the final timestep of the LSTM and pass it through a softmax for classification: $r^{\text {sent }}=r_{T}^{\text {forw }}$, and $\hat{S}=\operatorname{softmax}\left(W_{s} r^{\text {sent }}+b_{s}\right)$ where $r^{\text {sent }}$ is the sentence representation, and $\hat{S}$ is the label predicted. In our experiments with LSTM, we used 300-dimensional Glove embeddings to initialize the embedding layer for all the methods, e.g., baselines 1, 2 and our proposed compression method. Our vocabulary size $V$ contains only the words that appear in the training data, and we compress the input word embedding matrix $W$ (original size $V \times 300$ ).

Deep Averaging Network (DAN) DAN is a bag-of-words neural model that averages the word embeddings in each input utterance to create a sentence representation $r^{\text {sent }}$ that is passed through a series of fully connected layers, and fed into a softmax layer for classification. Assume an input sentence $X$ of length $T$ and corresponding word embeddings $e_{i}$, then the sentence representation is: $r^{\text {sent }}=\frac{1}{T} \sum_{i=1}^{T} e_{i}$

In our setup, we have two fully connected layers after the sentence representation $r^{\text {sent }}$, of sizes 1024 and 512 respectively. For the DAN experiments we used the DAN-RAND (Iyyer et al. 2015) variant where we take a randomly initialized 300 dimensional embedding. To make the comparison fair, all experiments with DAN models, including quantization (baseline 1), the offline compression method (baseline 2 ) and the proposed compression method are done using randomly initialized word embedding matrices. For example, our baseline 2 is to use appropriate low-rank randomly initialized embedding matrix.

\subsection{Experimental Setup}

Our experiments are performed on 1 Tesla K80 GPU, using SGD optimizer with CALR policy (Sec.3.2), initial learning rate upper bound of 0.001 . We use dropout of 0.4 between layers and L2 regularization with weight of 0.005 . Our metrics include accuracy and model size. Accuracy is simply the fraction of utterances with correctly predicted label. The model size is calculated as sum of (.data + .index.+ meta) files stored by tensorflow.

\subsection{Results on Uncompressed Models}

To effectively train our uncompressed benchmark networks we experimented with different adaptive learning rate schedules. Table 1 emperically demonstrates the effectiveness of our proposed learning rate update policy (CALR) on the SST2 test set. For both the models CALR outperforms the corresponding state-of-the-art sentence classification accuracy reported in the literature. For LSTM we improve the accuracy from $84.9 \%$ (Socher et al. 2013) to $86.03 \%(1.33 \%$ relative improvement) whereas on DAN-RAND we are able to improve from $83.2 \%$ (Iyyer et al. 2015) to $84.61 \%(1.7 \%$ relative improvement). We also report results on training the network with CLR without our cyclic annealing. For both DAN-RAND and LSTM, CLR achieves performance that is similar to the previously reported state-of-the-art. The proposed CALR outperforms CLR, which supports the effectiveness of the proposed CALR policy.

\subsection{Results on SST2}

Table 2 shows compression and accuracy results on the SST2 test dataset for our proposed methods, the two baselines described in Section 3.3, and for two types of models: LSTM (upper table rows) and DAN-RAND (lower table rows). For both types of models, we first report the original uncompressed model size and accuracy. For the quantization baseline (baseline 1), we report size and accuracy numbers for 8-bit and 16-bit quantization. For the SVD-based methods, both for the offline embedding compression (baseline 2) and our proposed compression method, we compare size and accuracy for different percentages of model size reduction $R$, where $R=1-p$. This reduction corresponds to different fractions $p$ and to different number of selected singular values $k$ (Section 3.1).

From Table 2, we observe that our proposed compression method outperforms both baselines for both types of mod-

\begin{tabular}{lll}
\hline Model & LR Schedule & Acc. $(\%)$ \\
\hline \multicolumn{3}{c}{ Uncompressed LSTM Model } \\
\hline Tai, Socher, and Manning & AdaGrad & 84.90 \\
Smith & CLR & 84.71 \\
Ours & CALR & $\mathbf{8 6 . 0 3}$ \\
\hline \multicolumn{3}{c}{ Uncompressed DAN-RAND Model } \\
\hline Iyyer et al. & AdaGrad & 83.20 \\
Smith & CLR & 83.84 \\
Ours & CALR & $\mathbf{8 4 . 6 1}$
\end{tabular}

Table 1: Comparison of initial Uncompressed Model Accuracy for different Learning Rate Schedules on SST2 


\begin{tabular}{|c|c|c|c|c|}
\hline Model & $\mathrm{R}(\%)$ & Size(MB) & $\operatorname{Acc}(\%)$ & $\operatorname{Acc}(\%)$ \\
\hline \multicolumn{5}{|c|}{ Uncompressed LSTM model } \\
\hline LSTM & $\mathbf{0}$ & 53.25 & 86.03 & - \\
\hline \multicolumn{5}{|c|}{ Quantized model (Baseline 1) } \\
\hline 16 bit & 50 & 30.16 & 85.08 & - \\
\hline 8 bit & 75 & 18.21 & 85.01 & - \\
\hline \multicolumn{5}{|c|}{ SVD: Proposed vs Offline Compression (Baseline 2) } \\
\hline \multirow{6}{*}{ LSTM } & & & Proposed & Baseline2 \\
\hline & 10 & 48.66 & 85.72 & 85.45 \\
\hline & 30 & 38.52 & 85.68 & 84.95 \\
\hline & 50 & 28.45 & 85.67 & 84.24 \\
\hline & 70 & 18.38 & 85.45 & 83.09 \\
\hline & 90 & 6.94 & 85.11 & 82.54 \\
\hline \multicolumn{5}{|c|}{ Uncompressed DAN model } \\
\hline DAN & $\mathbf{0}$ & 52.84 & 84.61 & - \\
\hline \multicolumn{5}{|c|}{ Quantized model (Baseline 1) } \\
\hline 16 bit & 50 & 28.36 & 83.18 & - \\
\hline 8 bit & 75 & 18.13 & 82.94 & - \\
\hline \multicolumn{5}{|c|}{ SVD: Proposed vs Offline Compression (Baseline 2) } \\
\hline \multirow{6}{*}{ DAN } & & & Proposed & Baseline2 \\
\hline & 10 & 47.29 & 84.24 & 83.47 \\
\hline & 30 & 37.23 & 83.83 & 83.31 \\
\hline & 50 & 27.16 & 83.72 & 82.87 \\
\hline & 70 & 17.18 & 83.67 & 82.86 \\
\hline & 90 & 6.21 & 83.11 & 82.59 \\
\hline
\end{tabular}

Table 2: Compression and accuracy results on SST2 dataset. $\mathrm{R}(\%)$ refers to percentage of model size reduction, where $R=1-p$, Size is the model size in $\mathrm{MB}$, and Acc is the classification accuracy(\%). All DAN models use the DANRAND variant.

els. For LSTM, our method achieves $R=90 \%$ compression with 85.11 accuracy e.g., only $1 \%$ rel. degradation compared to the uncompressed model, while the offline embedding compression (baseline 2) achieves 82.54 (4\% rel degradation vs uncompressed). Looking at $R=50 \%$ our method achieves accuracy of 85.67 (0.4\% rel degradation) vs 84.24 for baseline 2 (2\% rel degradation) and 85.08 for baseline 1 which does 16-bit compression (1\% rel degradation). For DAN-RAND and for $R=90 \%$ compression, our method achieves accuracy of 83.11 which corresponds to $1.7 \%$ rel degradation vs an uncompressed model, and outperforms baseline 2 that has accuracy of 82.59 (2.4\% rel. degradation). Similar trends are seen across various size reduction percentages $R$.

Comparing LSTM vs DAN-RAND models in terms of the gain we achieve over quantization, we observe that for LSTM our compression outperforms quantization by a large margin for the same compression rates, while for DANRAND the gain is less pronounced. We hypothesize that this is because LSTMs have recurrent connections and a larger number of low precision multiplications compared to DAN, which has been shown to lead to worse performance(Lin et al. 2015). Thus, we expect our method to benefit the most over quantization when the network is deep.

To illustrate how our proposed method benefits from retraining the model after compressing the embedding input matrix, in Fig 3 we plot the dev set accuracy for SST2 across training mini-batches for different compression percentages

\begin{tabular}{|c|c|c|c|c|}
\hline Model & $\mathrm{R}(\%)$ & Size(MB) & Acc. & Acc. \\
\hline \multicolumn{5}{|c|}{ Uncompressed LSTM model } \\
\hline LSTM & $\mathbf{0}$ & 29.34 & 91.78 & - \\
\hline \multicolumn{5}{|c|}{ Quantized model (Baseline 1) } \\
\hline 16 bit & 50 & 17.92 & 90.16 & - \\
\hline 8 bit & 75 & 10.05 & 89.96 & - \\
\hline \multicolumn{5}{|c|}{ SVD: Proposed vs Offline Compression (Baseline 2) } \\
\hline \multirow{6}{*}{ LSTM } & & & Proposed & Baseline2 \\
\hline & 10 & 26.76 & 91.71 & 91.07 \\
\hline & 30 & 21.69 & 91.63 & 90.97 \\
\hline & 50 & 16.62 & 91.54 & 90.87 \\
\hline & 70 & 10.31 & 91.47 & 90.58 \\
\hline & 90 & 4.89 & 90.94 & 89.83 \\
\hline \multicolumn{5}{|c|}{ Uncompressed DAN model } \\
\hline DAN & $\mathbf{0}$ & 37.03 & 90.18 & - \\
\hline \multicolumn{5}{|c|}{ Quantized model (Baseline 1) } \\
\hline 16 bit & 50 & 25.68 & 89.38 & - \\
\hline 8 bit & 75 & 16.41 & 88.86 & - \\
\hline \multicolumn{5}{|c|}{ SVD: Proposed vs Offline Compression (Baseline 2) } \\
\hline \multirow{6}{*}{ DAN } & & & Proposed & Baseline2 \\
\hline & 10 & 33.96 & 90.15 & 87.35 \\
\hline & 30 & 29.43 & 90.12 & 87.34 \\
\hline & 50 & 23.16 & 89.47 & 87.14 \\
\hline & 70 & 18.21 & 89.55 & 86.15 \\
\hline & 90 & 13.08 & 89.23 & 83.72 \\
\hline
\end{tabular}

Table 3: Compression and accuracy results on the Books Intent dataset. $\mathrm{R}(\%)$ refers to percentage of model size reduction, Size is the model size, and Acc is the classification accuracy. All DAN models use the DAN-RAND variant.

$\mathrm{R}$ and for both LSTM and DAN-RAND. For comparison, we plot the dev accuracy of 8-bit and 16-bit quantization (baseline 1) that remains stable across epochs as the quantized model is not retrained after compression. As a further comparison, we plot the uncompressed model accuracy on the dev which is kept stable as well (we assume this as our benchmark point). We observe that, while our compressed model starts off at a lower accuracy compared to both the uncompressed model and the quantization baseline, it rapidly improves with training and stabilizes at an accuracy that is higher than the quantization method and comparable to the original uncompressed model. This indicates that re-training after SVD compression enables us to fine-tune the layer parameters $W_{a}$ and $W_{b}$ towards the target task, and allows us to recover most of the accuracy.

\subsection{Results on Books Intent Dataset}

Table 3 shows compression and accuracy results on the proprietary Books Intent test set for our proposed methods vs the two baselines, for the LSTM and DAN-RAND models. Overall, we make similar observations as for the SST2 dataset. Our proposed method achieves better accuracy across compression percentages compared to both offline embedding compression and quantization for both LSTM and DAN-RAND. For example, for LSTM and R=90\% compression we achieve accuracy of $90.94 \%$ which corresponds to $1 \%$ degradation compared to the uncompressed model, while offline embedding compression (baseline 2) achieves accuracy of 89.83 ( $2 \%$ degradation). We also examined the 


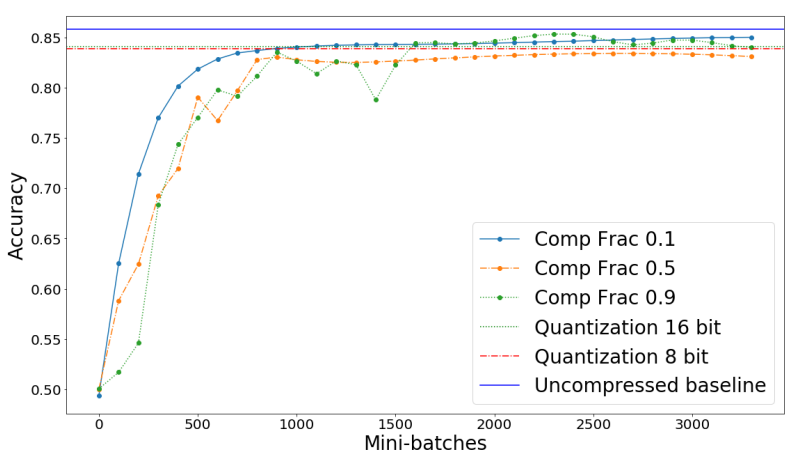

(a) LSTM model

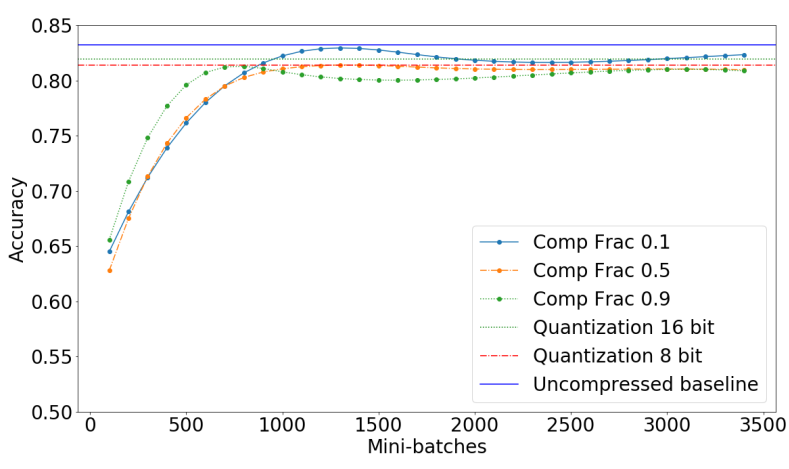

(b) DAN-RAND model

Figure 3: Dev set accuracy vs training mini batches for various compression percentages R for the SST2 dataset

\begin{tabular}{lllll}
\hline & LSTM & & DAN-RAND \\
\hline $\mathrm{R}(\%)$ & Proposed & Baseline 2 & Proposed & Baseline 2 \\
\hline 10 & 10.71 &.- & 1.38 & - \\
50 & 9.28 & 9.23 & 0.89 & 0.93 \\
70 & 9.21 &.- & 0.62 & - \\
75 & 9.11 & 9.08 & 0.59 & 0.62 \\
90 & 8.46 & - & 0.45 & - \\
\hline
\end{tabular}

Table 4: Inference Time on the SST2 test set (seconds)

plots of dev set accuracy while re-training the proposed compressed model across mini-batches and observed similar trends as for SST2 (plots are omitted for brevity).

\subsection{Results on Inference Time}

In Table 4, for both LSTM and DAN-RAND, we report inference times for the SST2 test set for different compression fractions using the proposed compression vs inference times for 16-bit and 8-bit fixed point quantization (baseline 2). As expected, for our method we see a decrease in inference time as the compression fraction $(\mathrm{R})$ increases. We observe similar trends for the quantization method, where 8-bit is faster than 16-bit. For similar compression rates (16-bit equivalent to $50 \%$ compression and 8-bit is equivalent to $75 \%$ compression) our method and baseline 2 (fixed-point-quantization) show similar inference times. Therefore, our method does not introduce any significant latency during inference while regaining the accuracy.

\section{Conclusions and Future Work}

In this work, we have proposed a neural model compression method based on low rank matrix factorization that can reduce the model memory footprint by an arbitrary proportion, that can be decided based on accuracy-memory trade-offs. Our method consists of compressing the model and then retraining it to recover accuracy while maintaining the reduced size. We have evaluated this approach on text classification tasks and showed that we can achieve up to $90 \%$ model size compression for both LSTM and DAN models, with minimal accuracy degradation (1\%-2\% relative) compared to an uncompressed model. We also showed that our method em- pirically outperforms common model compression baselines such as fixed point quantization and offline word embedding compression for the classification problems we examined. We have also provided an analysis of our method's effect on the model inference time and showed under which conditions it can achieve faster inference compared to model quantization techniques. An additional contribution of this work is the introduction of a novel learning rate scheduling algorithm, the Cyclically Annealed Learning Rate (CALR). We compared CALR to other popular adaptive learning rate algorithms and showed that it leads to better performance on the SST2 benchmark dataset. In future, we plan to evaluate the proposed compression method and the proposed CALR schedule on a larger variety of NLP tasks including sequence tagging and sequence to sequence modeling.

\section{References}

Anwar, S.; Hwang, K.; and Sung, W. 2015. Fixed point optimization of deep convolutional neural networks for object recognition. In ICASSP , 2015 IEEE International Conference on, 1131-1135. IEEE.

Bengio, Y.; Léonard, N.; and Courville, A. 2013. Estimating or propagating gradients through stochastic neurons for conditional computation. arXiv preprint arXiv:1308.3432.

Bergstra, J., and Bengio, Y. 2012. Random search for hyperparameter optimization. Journal of Machine Learning Research 13(Feb):281-305.

Bojanowski, P.; Grave, E.; Joulin, A.; and Mikolov, T. 2016. Enriching word vectors with subword information. arXiv preprint arXiv:1607.04606.

Chung, J.; Gulcehre, C.; Cho, K.; and Bengio, Y. 2014. Empirical evaluation of gated recurrent neural networks on sequence modeling. In NIPS 2014 Workshop on Deep Learning, .

Courbariaux, M.; Bengio, Y.; and David, J. 2014. Low precision arithmetic for deep learning. CoRR,abs/1412.7024 4.

Dauphin, Y. N.; Pascanu, R.; Gulcehre, C.; Cho, K.; Ganguli, S.; and Bengio, Y. 2014. Identifying and attacking the saddle point problem in high-dimensional non-convex optimization. In Advances in NIPS, 2933-2941. 
Duchi, J.; Hazan, E.; and Singer, Y. 2011. Adaptive subgradient methods for online learning and stochastic optimization. Journal of Machine Learning Research 12(Jul):21212159.

Golub, G. H., and Reinsch, C. 1970. Singular value decomposition and least squares solutions. Numerische mathematik 14(5):403-420.

Gong, Y.; Liu, L.; Yang, M.; and Bourdev, L. 2014. Compressing deep convolutional networks using vector quantization. arXiv preprint arXiv:1412.6115.

Han, S.; Pool, J.; Tran, J.; and Dally, W. 2015. Learning both weights and connections for efficient neural network. In Advances in NIPS, 1135-1143.

Han, S.; Mao, H.; and Dally, W. J. 2015. Deep compression: Compressing deep neural networks with pruning, trained quantization and huffman coding. arXiv preprint arXiv:1510.00149.

Hinton, G.; Vinyals, O.; and Dean, J. 2015. Distilling the knowledge in a neural network. arXiv preprint arXiv:1503.02531.

Hochreiter, S., and Schmidhuber, J. 1997. Long short-term memory. Neural computation 9(8):1735-1780.

Hubara, I.; Courbariaux, M.; Soudry, D.; El-Yaniv, R.; and Bengio, Y. 2016. Quantized neural networks: Training neural networks with low precision weights and activations. arXiv preprint arXiv:1609.07061.

Iyyer, M.; Manjunatha, V.; Boyd-Graber, J.; and III, H. D. 2015. Deep unordered composition rivals syntactic methods for text classification. Proceedings of the 53rd Annual Meeting of the ACL.

Joulin, A.; Grave, E.; Bojanowski, P.; Douze, M.; Jégou, H.; and Mikolov, T. 2016. Fasttext. zip: Compressing text classification models. arXiv preprint arXiv:1612.03651.

Keskar, N. S.; Mudigere, D.; Nocedal, J.; Smelyanskiy, M.; and Tang, P. T. P. 2016. On large-batch training for deep learning: Generalization gap and sharp minima. arXiv preprint arXiv:1609.04836.

Kim, Y. 2014. Convolutional neural networks for sentence classification. In Proc. of the 2014 Conference on EMNLP.

Kingma, D. P., and Ba, J. 2014. Adam: A method for stochastic optimization. arXiv preprint arXiv:1412.6980.

Lewicki, M. S., and Sejnowski, T. J. 2000. Learning overcomplete representations. Neural computation 12(2):337365.

Lin, Z.; Courbariaux, M.; Memisevic, R.; and Bengio, Y. 2015. Neural networks with few multiplications. arXiv preprint arXiv:1510.03009.

Liu, P.; Qiu, X.; Chen, X.; Wu, S.; and Huang., X. 2015. Multi-timescale long short-term memory neural network for modelling sentences and documents. In In Proceedings of the Conference on EMNLP 2015.

Loshchilov, I., and Hutter, F. 2016. Sgdr: Stochastic gradient descent with warm restarts. arXiv preprint arXiv:1608.03983.
Lu, Z.; Sindhwani, V.; and Sainath, T. N. 2016. Learning compact recurrent neural networks. In ICASSP , 2016 IEEE International Conference on, 5960-5964. IEEE.

Ma, X., and Hovy, E. 2016. End-to-end sequence labeling via bi-directional lstm-cnns-crf. In Proc. of the 54th Annual Meeting of the ACL 2016.

Mikolov, T.; Chen, K.; Corrado, G.; and Dean, J. 2013. Efficient estimation of word representations in vector space. arXiv preprint arXiv:1301.3781.

Pennington, J.; Socher, R.; and Manning, C. D. 2014. Glove: Global vectors for word representation. In EMNLP, 15321543.

Polino, A.; Pascanu, R.; and Alistarh, D. 2018. Model compression via distillation and quantization. arXiv preprint arXiv: 1802.05668.

Raunak, V. 2017. Effective dimensionality reduction for word embeddings. arXiv preprint arXiv:1708.03629.

Recht, B., and Ré, C. 2013. Parallel stochastic gradient algorithms for large-scale matrix completion. Mathematical Programming Computation 5(2):201-226.

Sainath, T. N.; Kingsbury, B.; Sindhwani, V.; Arisoy, E.; and Ramabhadran, B. 2013. Low-rank matrix factorization for deep neural network training with high-dimensional output targets. In ICASSP , 2013 IEEE International Conference on, 6655-6659. IEEE.

See, A.; Luong, M.-T.; and Manning, C. D. 2016. Compression of neural machine translation models via pruning. arXiv preprint arXiv:1606.09274.

Shu, R., and Nakayama, H. 2017. Compressing word embeddings via deep compositional code learning. arXiv preprint arXiv:1711.01068.

Smith, L. N. 2017. Cyclical learning rates for training neural networks. In Applications of Computer Vision (WACV), 2017 IEEE Winter Conference on, 464-472. IEEE.

Socher, R.; Perelygin, A.; Wu, J.; Chuang, J.; Manning, C. D.; Ng, A.; and Potts, C. 2013. Recursive deep models for semantic compositionality over a sentiment treebank. In Proceedings of the 2013 conference on empirical methods in natural language processing, 1631-1642.

Tai, K. S.; Socher, R.; and Manning, C. D. 2015. Improved semantic representations from tree-structured long shortterm memory networks. arXiv preprint arXiv:1503.00075.

Trefethen, L. N., and Bau III, D. 1997. Numerical linear algebra, volume 50. Siam.

Wen, W.; Wu, C.; Wang, Y.; Chen, Y.; and Li, H. 2016. Learning structured sparsity in deep neural networks. In $A d-$ vances in NIPS, 2074-2082.

Xue, J.; Li, J.; and Gong, Y. 2013. Restructuring of deep neural network acoustic models with singular value decomposition. In Interspeech, 2365-2369.

Zhou, A.; Yao, A.; Guo, Y.; Xu, L.; and Chen, Y. 2017. Incremental network quantization: Towards lossless cnns with low-precision weights. arXiv preprint arXiv:1702.03044. 